\title{
FMIRS: A Fuzzy indexing and retrieval system of mosaic-image database
}

\author{
Maghrebi Wafa ${ }^{1}$, Khabou Mohamed A ${ }^{2}$, Alimi Adel M. ${ }^{1}$ \\ ${ }^{I}$ REGIM: REsearch Group on Intelligent Machines, University of Sfax, Tunisia \\ ${ }^{2}$ Electrical and Computer Engineering Dept, University of West Florida, USA \\ Received $31^{\text {st }}$ January 2014; accepted $27^{\text {th }}$ Oct 2014
}

\begin{abstract}
This work is dedicated to present a fuzzy-set based system useful for image indexing and retrieval pertaining to historical Roman-mosaics. This exceptional collection of mosaics dates back from the first to fourth centuries AD. Considering the state of these images (i.e. noise, color degradation, etc.) a fuzzy features definition is necessary. Thereby, we use a robust to rotation, scale and translation fuzzy extended curvature scale space (CSS) as shape descriptor. Furthermore, we propose a fuzzy color-quantization approach, applied on mosaics, using HSV color space. The system allows for two user-friendly querying modes: a drawing based mode and the mode that fusion both shape and color features using a unified fuzzy similarity measure. Based on queries of variable complexity, the advanced fuzzy system has managed to achieve interesting recall, precision and F-measure rates.
\end{abstract}

Key words: Fuzzy system, fuzzy color-quantization, fuzzy CSS shape descriptor, Roman mosaics, fuzzy similarity.

\section{Introduction}

A trend of museums creating digital showrooms/archives of the real paintings and artifacts they contain has emerged lately. The digital showrooms usually consist of high-resolution images of the paintings and artifacts devised to preserve the original pieces of work and make them available to the reach of a wider audience via the Internet. In this respect, Van den Broek et al. [1] have designed the C-BAR system (Content Based Art Retrieval) to index and present a digitized version of a $17^{\text {th }}$-century painting-collection belonging to the Netherlands' national gallery (the Rijksmuseum http://www.rijksmuseum.nl/). In this context Broek et al.[2] have continued with improving the system's performance and user interface. The Hermitage Museum, in collaboration with its partner IBM, presented by the QBIC (Query By Image Content) system (Flickner et al.[3]) that operates by applying layout and color queries to retrieve its stored digital-collection pertaining images (http://www.hermitagemuseum.org). As for (Schomaker et al.[4], Vuupijl et al.[5]), the authors have put forward the Vindx system as referential framework which helps index the digitized collection of the Rijksmuseum wide-array of images by allowing the user to specify a textual description of the object(s) he or she is trying to retrieve among the digitized images. The rationale advanced by the authors in favor of the object-based matching process lies in the fact that users are predominantly interested in the object content, rather than in the layout. Within the same line of thought, Chang and Kim [6] have considered, in their elaborated work, setting up a retrieval system useful for application to the digital of Korean-porcelain images. Actually, the authors have proposed introducing a fusion between normalizedcolor histogram and the centroid-distance vector as object-shape descriptors. The system uses high-resolution images consisting of mono-object porcelain artifacts, photographed on a uniform background.

\author{
Correspondence to: < wafa.maghrebi@fsegs.rnu.tn> \\ http://dx.doi.org/10.5565/rev/elcvia.608 \\ Recommended for acceptance by $<$ Xavier Otazu $>$ \\ ELCVIA ISSN: $1577-5097$ \\ Published by Computer Vision Center / Universitat Autonoma de Barcelona, Barcelona, Spain
}


With regard to the Tunisian context, the National Library (http://www.bibliotheque.nat.tn), the National Archives (http://www.archives.tn), and a selection of other museums (http://www.patrimoinedetunisie. com.tn) such as Bardo, El-Jem, Enfidha, Sousse and Sfax, contain a huge selection of ancient documents, mosaics, and artifacts of important historical value. These treasures are carefully photographed and catalogued in such a way to be at the reach of researchers, thus avoiding direct handling of such fragile items. Some researchers have recently tackled the problem of indexing and cataloguing mosaic-related images with all the challenges such image peculiarities could present (Stanco et al.[7], M'hedbi et al.[8], Maghrebi et al.[9]). In this respect, M'hedbi et al.[7] use a CBIR (Content Based Information Retrieval) approach, whereby to retrieve mosaics based on shape descriptors, while Maghrebi et al.[9] present a retrieval system of Roman-mosaic images using drawing queries, involving a robust MPEG7 shape descriptors to index objects. However, users (laypersons and experts) involved in the cultural-heritage domain usually want to describe an object they are looking for. Since we treat historical images, with all the deficiencies they represent (noise, degradation of color, etc.); a fuzzy system is advanced in this paper for the purpose of indexing and retrieving rich and complex mosaics (see figure1). The main contribution of this work is the introduction of color and its fusion with shape to retrieve mosaic-images. Noteworthy, also, the retrieval process proposes a two-querying-modes process designed to be as much user-friendly and simple to implement as possible while guaranteeing a maximum level of ease of use.

The remainder of this paper is organized as follows: the second section is dedicated to present the toplevel architecture of our designed scheme, while section 3 presents the mosaic-image database (DB) and the preprocessing step, followed by the section 4 which interests to present the fuzzy color quantization approach. As for section 5, it is devoted to describe the shape fuzzy features. Regarding the query and the fuzzy retrieval process, it is described in section 6, and the achieved experimental results are depicted in section 7 and last section is reserved to highlight the concluding remarks, research perspectives and horizons for future works.
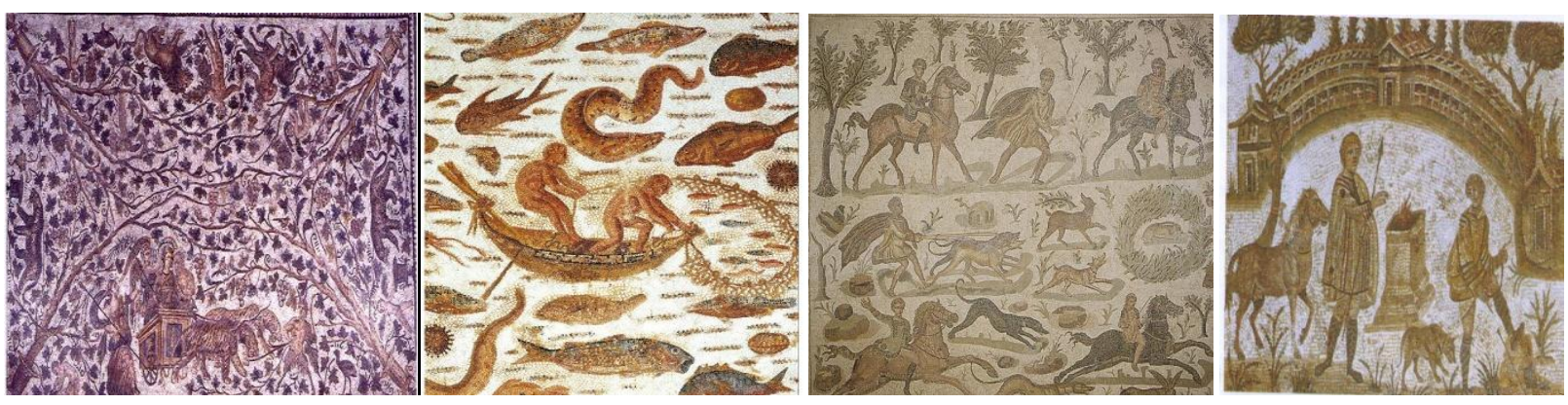

Figure1. Samples of Tunisian historical mosaics

\section{The system general architecture}

It is worth highlighting that our devised system's general architecture is depicted in figure 2, below. Once a mosaic input image is provided to the FMIRS system, the later would allow the user to specify/extract the relevant objects using an on-line annotation module, which relies on a user's perception to annotate areas of interest and important shapes/objects in a mosaic image. More details can be found in Maghrebi et al.[10]. By using an object boundary as a guiding outline, we extract, automatically, the objects' robust shape crisp descriptors. Our shape features are a combination of both global and local features. The global features consist of the circularity and eccentricity. The local ones are the definition of shape concavities and convexities to represent an extended version of the Curvature-Scale Space (CSS). More details regarding these features are depicted in section 5. In addition, the proposed approach suggests color-features extraction based on the use of a fuzzy quantized-HSV-color space from preprocessed mosaic-image DB. To note, fuzzy sets are defined according to the hue $(\mathrm{H})$, saturation $(\mathrm{S})$ and value $(\mathrm{V})$ components of the HSV color space, through which we attempt to provide a fuzzy logic model that aims at following the human intuition for color classification. The five most dominant colors (based on the normalized areas they occupy in respect of the targeted object) are determined and included within the image indexing process.

Noteworthy, however, these extracted fuzzy visual features are used in the retrieval process. The user can apply either drawing query or query that simultaneously combines the relevant object's color and boundary. Using a fuzzy similarity measure the system returns relevant images. 


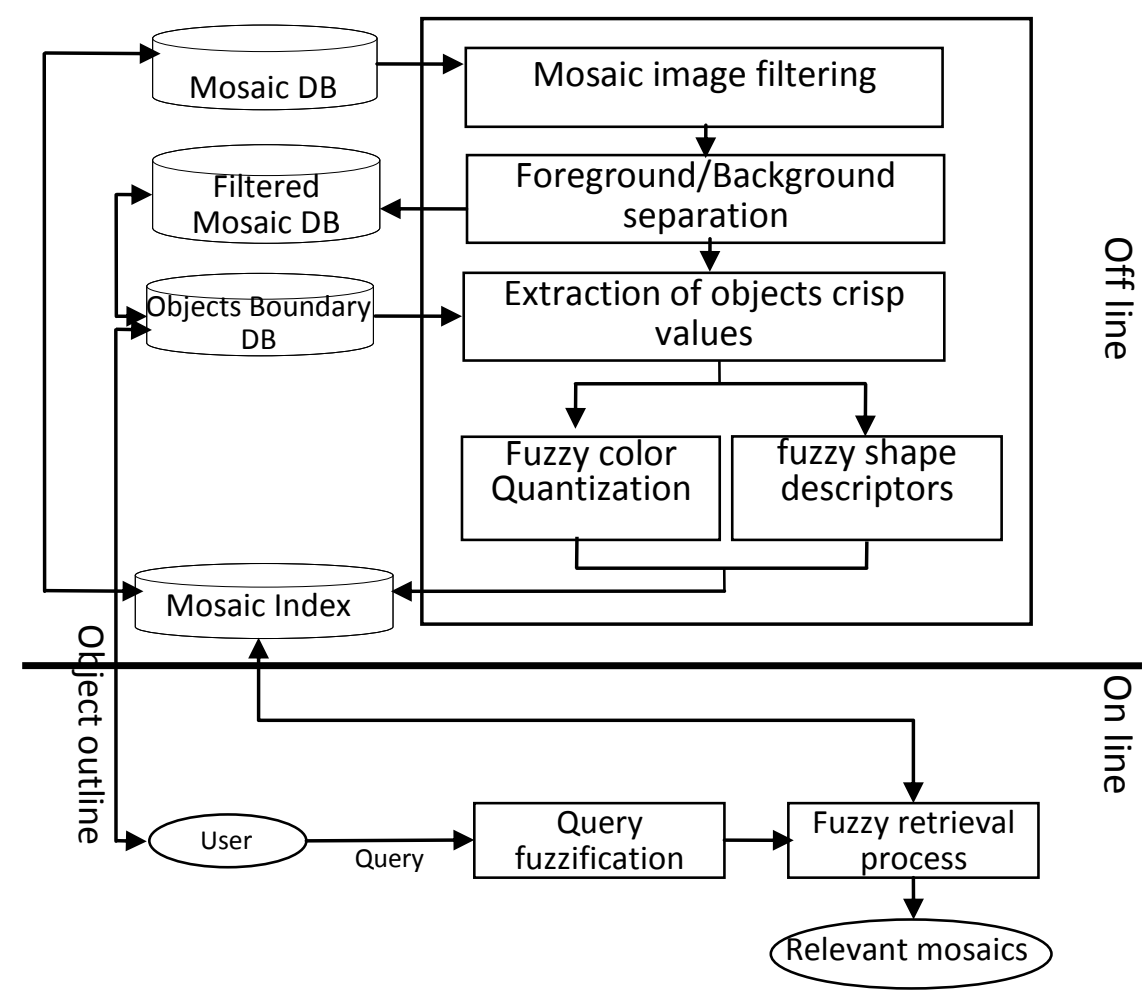

Figure2. The system general architecture

\section{Mosaic-images database preprocessing}

Mosaics are a harmony of marble tesserae. So, mosaics are of natural colors and complex content. These images are resized to 512x512 and have been filtered with a Gaussian and median filters to reduce the noise caused by the mosaic architecture and the brisk intensity variation respectively. Furthermore, we use the Huang and Wang [11] threshold approach for the background/foreground separation to take only relevant information from the image. The Huang and Wang thresholding approach is based on minimizing the measure of fuzziness of an input image. The membership function in the thresholding method is used to denote the characteristic relationship between a pixel and its belonging region (the object or the background). It defines the absolute difference between the pixel gray level and the average gray level of the belonging region. Let I denote an image set of size $\mathrm{Mx} \mathrm{N}$ with L levels and $f(x, y)$ is the gray level of a $(x, y)$ pixel in $\mathrm{I}$. So, the fuzzy membership function is defined by:

$$
\mu_{\mathrm{I}}(f(x, y))= \begin{cases}\frac{1}{1+\left|f(x, y)-m_{1}(T)\right| / D} & \text { if } f(x, y) \leq T \\ \frac{1}{1+\left|f(x, y)-m_{2}(T)\right| / D} & \text { if } f(x, y)>T\end{cases}
$$

With $f(x, y)$ represents the gray level of the pixel $(x, y)$, while the measures $m_{1}, m_{2}$ are defined based on the Threshold $T$ and histogram $h(z)$ of the gray level $z$. The respective equations of $m_{l}$ and $m_{2}$ are as follows:

$$
m_{1}(T)=\frac{\sum_{z=0}^{T} z h(z)}{\sum_{z=0}^{T} h(z)} \quad m_{2}(T)=\frac{\sum_{z=T+1}^{L-1} z h(z)}{\sum_{z=T+1}^{L-1} h(z)}
$$

$\mathrm{D}$ is a constant defined by the equation 3 as follows:

$$
D=z_{\max }-z_{\min }
$$

The main role is to find a membership degree between 0.5 and 1 in order to use Shannon function of Luca and Termini which define the entropy of fuzzy sets as: 


$$
E(T)=\frac{1}{\ln 2} \sum_{z=0}^{L-1} h(z) \operatorname{Se}\left(\mu_{I}(z)\right)
$$

Where $\mu_{\mathrm{I}}(\mathrm{z}) \in[0.5,1]$. With the Shannon function, $S e$ is defined as follows:

$$
\operatorname{Se}\left(\mu_{I}(z)\right)=-\mu_{I}(z) \ln \left(\mu_{I}(z)\right)-\left(1-\mu_{I}(z)\right) \ln \left(1-\mu_{I}(z)\right)
$$

We retain the optimal threshold value that corresponds to the minimum measure:

$$
T^{*}=\arg \max _{0 \leq T \leq L} E(T)
$$

Figure 3 shows examples of background/foreground separation using Huang and Wang approach.

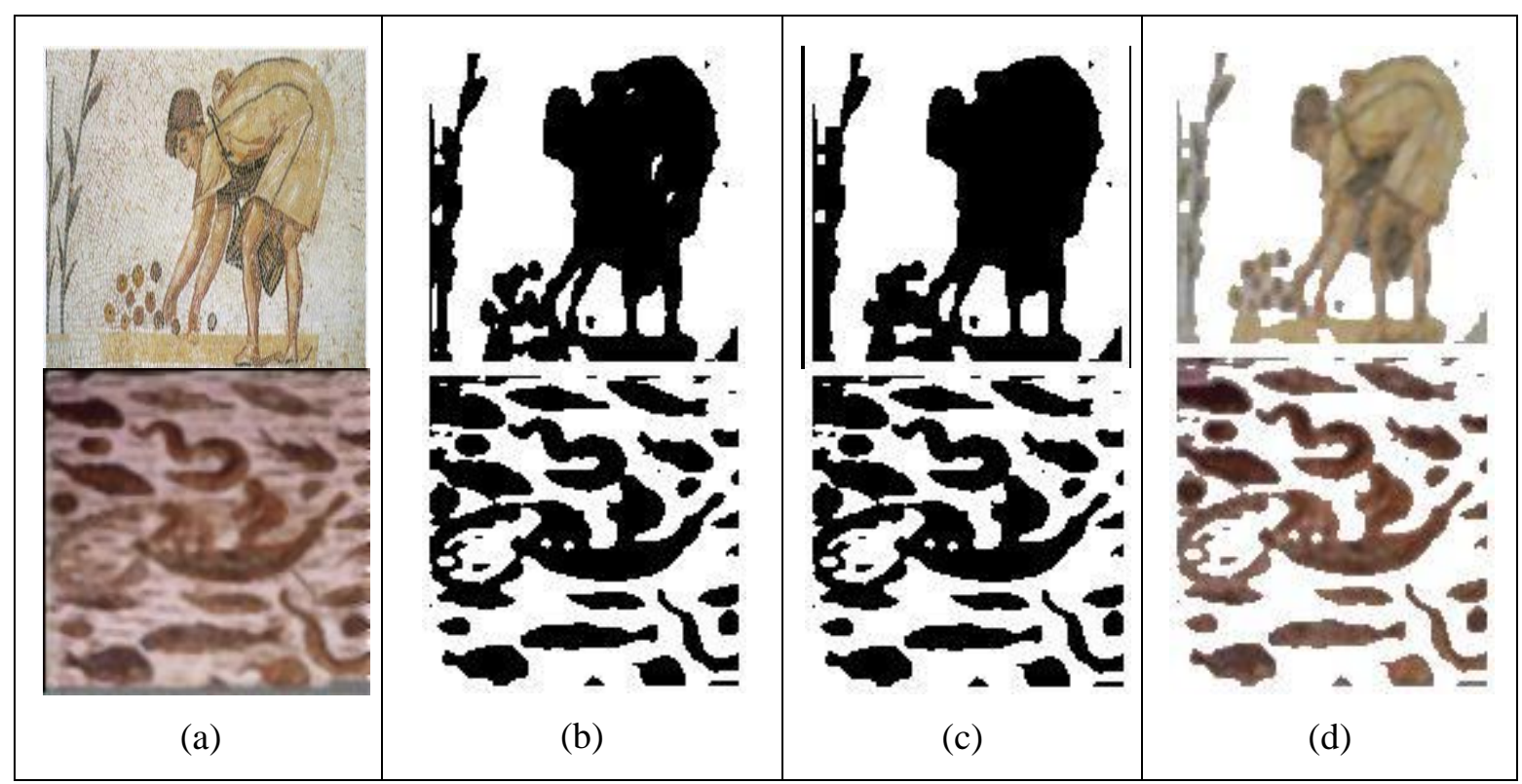

Figure 3. a) Original image; b) binary image using the Huang and Wang thresholding approach; c) application of morphological operators and d) mosaic-image foreground

\section{Presentation of the fuzzy-color naming approach}

\subsection{Related works}

Research dealing with color modeling for computational purposes has concentrated on seeking numerical color-representations fit for application to computer graphics and image processing. The problem is that none of these color spaces maps well onto the human perception of colors. Indeed, within a random set of RGB (Red, Green and Blue) or HSV values, even experienced humans can have difficulty determining the exact color representation is being depicted. (e.g what color is represented by the RGB triple $(0.5,0.1,0)$ or by the HSV triple $(0.03,1,0.5))$. The reverse process is perhaps even more complex as in the case with: what are the exact RGB or HSV triples that best represent the color "Brown". In this context, several studies have been conducted with the aim of creating color-semantic-labeling systems using a color space quantization (Berk et al.[12], Conway [13], Kelly[14], Shamir [15]). Berk et al.[12], with their devised Color Naming System (CNS), have propose to decipher 627 distinct colors, along with the designed ISCC-NBS system (Kelly[14]) using 267 different linguistic-color labeling based on color centroids, as well as the Conway system (Conway [13]) which proposes a quantized HSL color space distinguishing between 141 distinct colors. In turn, Shamir [15] proposes an image segmentation method based on fuzzy quantization HSV color space approach using ten, five and four fuzzy sets useful for representing the Hue, Saturation and Value fuzzy sets respectively. Benavente et al.[16] present the color-naming experiment developed to obtain a set of color judgments adequate for the fuzzy modeling of the color-naming task. Since, we use the historic mosaics (dates back from the $1^{\text {st }}$ to the $4^{\text {th }}$ century $\mathrm{AD}$ ) which are in a vulnerable situation and present a pale and degraded color, our purpose is in the sense to apply a fuzzy color-quantization to present the color-fuzziness of mosaics. 


\subsection{The mosaic color specification}

It should be noted that for the sake of extracting color descriptor, the HSV color space needs to be applied (rather than RGB space). Indeed, the HSV color space proves to be more intuitive and closer to the human perception than the RGB space. In fact, within an HSV-space, the color is depicted by its hue component $(\mathrm{H})$ with values ranging between 0 and 360, which actually code the color itself, the saturation (S) and value (V) with values comprised between 0 and 100. It is worth recalling that the last two HSV components refer to the color richness and brightness.

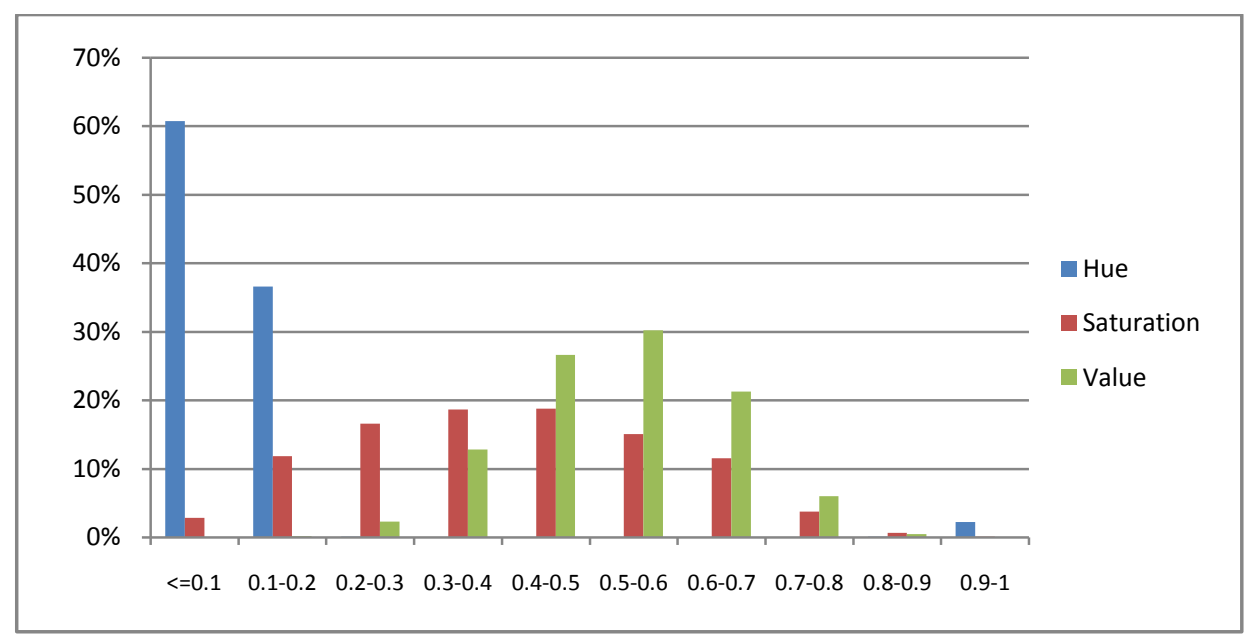

Figure 4. The HSV color-space distribution of the mosaic-images DB

It is worth mentioning that the historical mosaic-image database is with degraded colors. To study this thesaurus, we have removed the background using the Huang and Wang approach (see section 3) and on implementing the normalized components of the HSV color space, depicted in figure 4 and presented in table1, it has been discovered that:

- $99 \%$ of the hue component turns out to correspond to the red, orange and yellow spectra, divided as $63 \%$ for the red and orange spectra and 36\% for the orange and yellow spectra.

\begin{tabular}{|l|c|c|c|}
\hline \multirow{2}{*}{ Value } & \multicolumn{3}{|c|}{ Pixels distribution/ component } \\
\cline { 2 - 4 } & Hue & Saturation & Value \\
\hline$<=0.1$ & $60.756 \%$ & $2.842 \%$ & $0.007 \%$ \\
\hline $0.1-0.2$ & $36.639 \%$ & $11.842 \%$ & $0.194 \%$ \\
\hline $0.2-0.3$ & $0.159 \%$ & $16.602 \%$ & $2.303 \%$ \\
\hline $0.3-0.4$ & $0.015 \%$ & $18.679 \%$ & $12.838 \%$ \\
\hline $0.4-0.5$ & $0.019 \%$ & $18.779 \%$ & $26.622 \%$ \\
\hline $0.5-0.6$ & $0.008 \%$ & $15.104 \%$ & $30.225 \%$ \\
\hline $0.6-0.7$ & $0.028 \%$ & $11.569 \%$ & $21.292 \%$ \\
\hline $0.7-0.8$ & $0.017 \%$ & $3.782 \%$ & $6.050 \%$ \\
\hline $0.8-0.9$ & $0.104 \%$ & $0.671 \%$ & $0.467 \%$ \\
\hline $0.9-1$ & $2.255 \%$ & $0.129 \%$ & $0.001 \%$ \\
\hline
\end{tabular}

Table1. Statistics on HSV color space component applied on mosaics images 
- As highlighted by figure 4, 2.8\% of the pixels' saturation components are a variation of gray, while $47 \%$ as pale (i.e. the saturation values are between 0.1 and 0.4 ), 50\% are of pastel saturation (with values ranging between 0.4 and 0.9 ) and only $0.12 \%$ are discovered to be pure, with respect to the Conway classification (Conway[13]). Consequently, the Tunisian-mosaic images' database color turn out to be, predominantly, featured between the pale and pastel saturation sets.

- Based on the Conway classification (Conway [13]), the value component focus mainly on dark colors, as seen in table 1 . In fact, $2.5 \%$ of mosaic-images pixels are of very_dark value variation whereas $91 \%$ pixels are in the interval $[0.3,0.7]$ and only $6.5 \%$ ones are between 0.7 and 1.

\subsection{The mosaic fuzzy-color quantization method}

It is worth reminding that color-features quantization process constitutes a key feature with regard to retrieval systems. It is mainly applied to support semantically based image retrieval as a more effective, and widely used, measure to quantize color based on color labeling. Consequently, we reckon to apply a set of simple semantic adjectives to designate the saturation, following the terms undertaken by Conway, namely: pale, pastel and pure, while inserting the gray saturation-level to the semantic-saturation set. So that it becomes as a set of: gray, pale, pastel and pure. In addition, we have undertaken to extend the "pure" interval, defined by Conway to be comprised between 0.9 and 1, to include saturation values ranging between 0.8 and 1 . The value-component is decomposed into three semantic adjectives which are: verydark, dark and light. We also reckon to extend the light interval to reach luminance values comprised between 0.7 and 1 , instead of values contained between 0.8 and 1 as in the Conway classification case. To note, the hue component is mainly concentrated within the interval of red/orange/yellow color-variation. So, to handle the entirety of these colors' variation, we propose to decompose this color-spectrum range by defining two other colors, namely, the dark_orange and light_orange, as we reckon the brown color to be automatically formed by the value variation. Thus, we suggest decomposing the hue component into 10 basic colors: red, dark_orange, orange, light_orange, yellow, green, aqua, blue, purple and pink. In this study, the proposed decomposition would yield closely tied intervals pertinent to the red, orange and yellow colors (i.e. to set up various brown-color degradation), along with wide intervals for the other colors based on their color centroids. For illustration purposes, Figure 5 highlights the Hue variations attained via, our devised, Hue fuzzy-classification. This classification scatters the $97 \%$ of mosaic pixels (see figure 4 ) into five intervals from the red, yellow_red and yellow spectra based on the Munsell color representation.

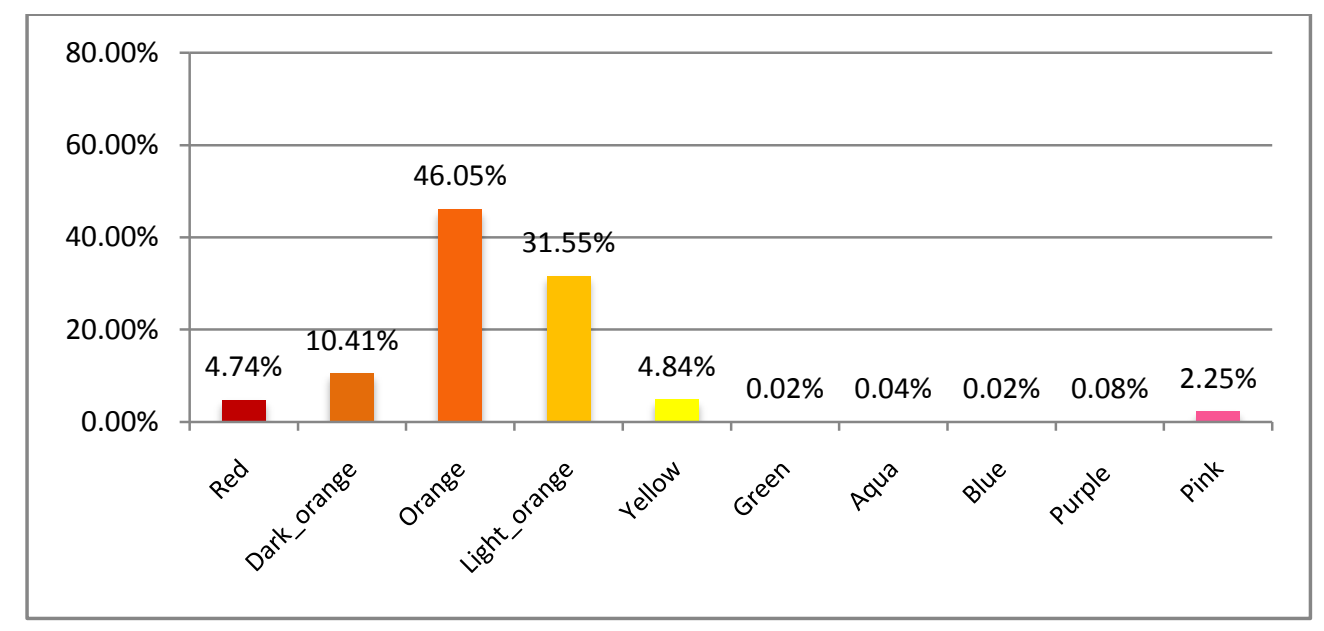

Figure 5. The Hue components' repartition respect to the predefined fuzzy sets

Noteworthy, also, the triangle function has been used with regard to Hue and saturation component. Figures $6 \mathrm{a}$ and $6 \mathrm{~b}$ respectively show the Hue as well as the Saturation-level fuzzy sets. Whereas, the value component is represented by the trapezoidal and triangle functions as represented in figure $6 \mathrm{c}$. In this way 
each HSV component turns out to be fuzzified in respect of their membership degree to the corresponding membership functions.

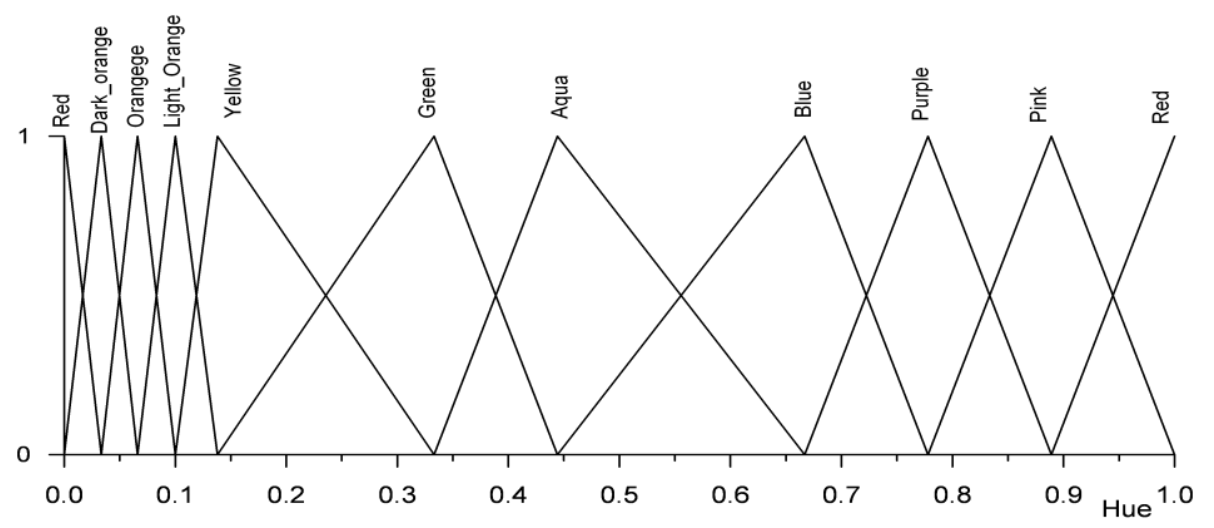

(a)

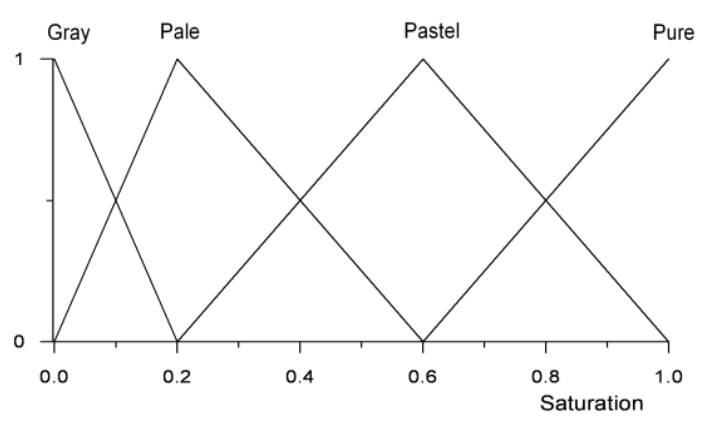

(b)

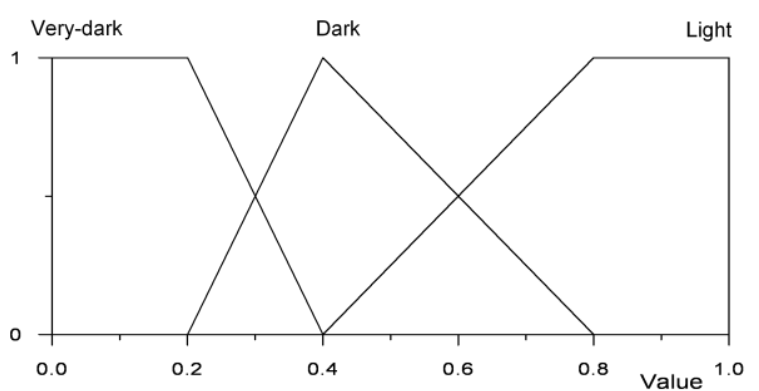

(c)

Figure 6. Fuzzy sets of the components a) Hue b) Saturation c) value

Thus, on the basis of a certain object's on the H, S, and V membership degrees, a set of $10 \times 4 \times 3=120$ rules proves to be discovered, enabling to determine and define ,more accurately, what a human would perceive as an object's dominant color. For a further clarification of such a scheme idea, let's, for instance, consider the following sample: if $\mathrm{H}=15, \mathrm{~S}=100$ and $\mathrm{V}=40$, the automatically-developed fuzzy inference process would discover a dark_brown color exposed together with its different relevant fuzzy sets in the forms of: hue is dark_orange, saturation is pure and value is dark. Moreover, for every Hue value with gray in the Saturation level and light in the Value one the color turns out to be white. This process is applied using the NBS-ISCC Color naming dictionary ${ }^{1}$ to define 120 colors. In table 2 we present some samples of fuzzy color naming based on HSV components fuzzy linguistic terms.

\begin{tabular}{|l|l|l|l|}
\hline \multicolumn{1}{|c|}{ Hue } & \multicolumn{1}{c|}{ Saturation } & \multicolumn{1}{c|}{ Value } & \multicolumn{1}{c|}{ Color naming } \\
\hline dark orange & Pure & Dark & Dark Brown \\
\hline orange & Pure & Dark & Brown \\
\hline yellow & Pure & Light & Yellow \\
\hline Green & Pure & Dark & Green \\
\hline Green & Pure & Light & Light Green \\
\hline- & Gray & Very dark & Black \\
\hline- & Gray & Dark & Gray \\
\hline- & Gray & Light & White \\
\hline
\end{tabular}

Table2. Samples of fuzzy-color naming

\footnotetext{
${ }^{1}$ http://people.csail.mit.edu/jaffer/Color/Dictionaries
} 
Note that the fuzzy rules relevant evaluation has been performed by means of fuzzy-set operations. In this regard, MIN and MAX operations have been applied for respectively the "AND" and "OR" operators. After evaluating each-rule's activation degree we have undertaken to up for the maximum algorithm as an accumulation method. As for the object-indexing procedure, it has been performed by using the first five dominant colors. Figure 7 illustrates some samples of returned object colors based on predefined fuzzy rules, whose membership degrees and normalized occupied area have also been saved.

\begin{tabular}{|c|c|}
\hline 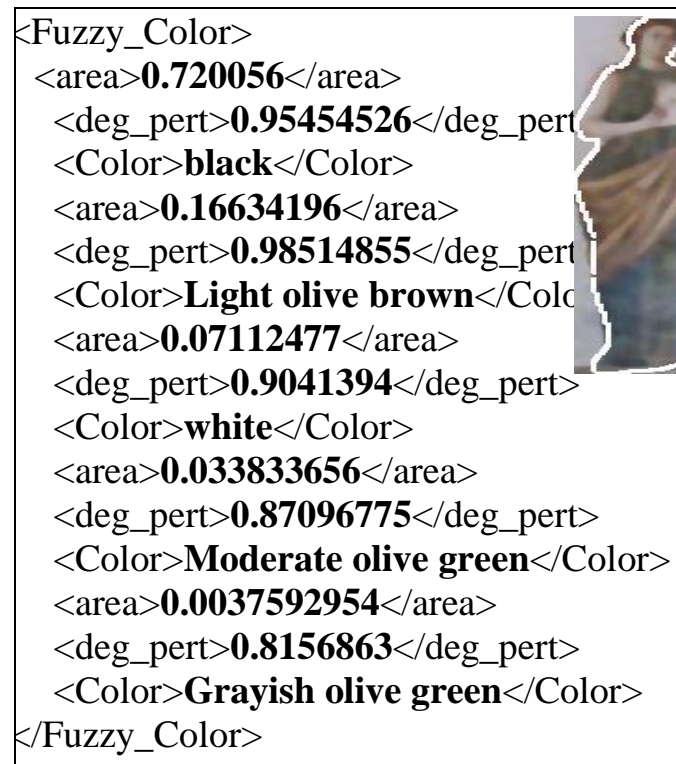 & 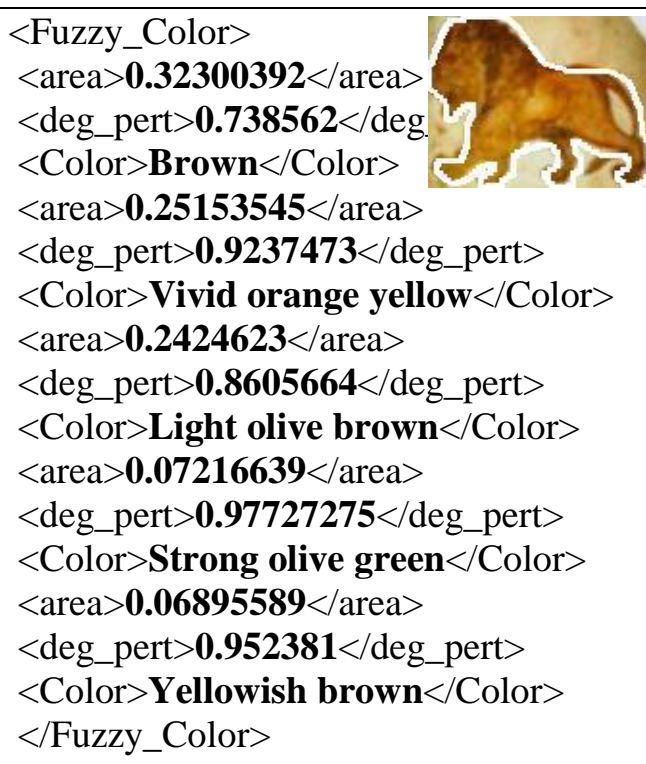 \\
\hline
\end{tabular}

Figure7. Samples of object fuzzy quantized-color

\section{Extraction of the shape features}

\subsection{The robust-shape index}

Initially introduced by Mokhtarian et al.[17], the Curvature Scale Space (CSS) descriptors help to register a curve pertinent concavities while undergoing a successive filtering process. In fact the role of the filtering procedures lies in smoothing out the curve using a Gaussian kernel $g(u, \sigma)$ and gradually eliminating the concavities appearing with an increasing size. The staking of a succession of inflexions points resulting from the filtering iteration construct the CSS image. More details can be found in the reference Maghrebi et al.[9].

The returned image represents, as abscissa, the curvilinear abscissa $\mu$ and, as ordinate, the number of filtering iteration $\sigma$. Our CSS index is represented by the peaks $\left(\mu_{\mathrm{n}}, \sigma_{\mathrm{n}}\right)$ relevant to the CSS image, with $\sigma_{\mathrm{n}}>\sigma_{\mathrm{th}}$ . Where $\sigma_{\text {th }}$ is the filtering threshold at which the object boundary noise is eliminated).

Object_CSS $=\left\{\left(u_{1}, \sigma_{1}\right),\left(u_{2}, \sigma_{2}\right),\left(u_{3}, \sigma_{3}\right),\left(u_{4}, \sigma_{4}\right), \ldots,\left(u_{n}, \sigma_{n}\right)\right\}$ with $\sigma_{i}<\sigma_{\text {th }} i \in[1, n]$

These peaks are sorted in a decreasing order of $\sigma$, as illustrated by the following example:

Object_CSS $=\{(0.00,20.0)(0.63,17.8)(0.23,8.6)(0.02,8.6)(0.40,8.3)(0.63,8.3)(0.82,6.8)(0.96,5.2)\}$

Even though the CSS descriptors have the advantage of being invariant to scale, translation, and rotation, as they prove to be robust and tolerant of noise, they turn out to be inadequate to represent a certain shape's convex segments. In this respect, Kopf et al. [18] presented a solution whereby to remedy the CSS descriptors' failure to represent convex segments of a shape. The idea they have put forward consists of constructing a dual shape relevant to the input shape, in which the convex segments, in their entirety, are transformed to concave segments. The dual shape is created by mirroring the input shape with respect to the circle of a minimum radius $R$ that encloses the original shape. More precisely, each point $(x(u), y(u))$ of the original shape is paired with a point $\left(x^{\prime}(u), y^{\prime}(u)\right)$ of the dual shape in such a way that the distance from $(x(u), y(u))$ to the circle is similar to that from $\left(x^{\prime}(u), y^{\prime}(u)\right)$ to the circle. The equations 7 and 8 define the projected point coordinates $\left(x^{\prime}(u), y^{\prime}(u)\right)$, which is located at: 


$$
\begin{gathered}
x^{\prime}(u)=\frac{2 R-D_{x(u), y(y)}}{D_{x(u), y(y)}}\left(x(u)-M_{x}\right)+M_{x} \\
y^{\prime}(u)=\frac{2 R-D_{x(u), y(y)}}{D_{x(u), y(y)}}\left(y(u)-M_{y}\right)+M_{y}
\end{gathered}
$$

Where $\mathrm{D}_{\mathrm{x}(u), y(u)}$ stands for the distance between the circle centre and the original shape pixel. $\mathrm{M}_{\mathrm{x}}$ and $\mathrm{M}_{\mathrm{y}}$ are the coordinates of the circle's centre $O\left(M_{x}, M_{y}\right)$, which are calculated in the following way:

$$
M_{x}=\frac{1}{N} \sum_{u=1}^{N} x(u) \quad M_{y}=\frac{1}{N} \sum_{u=1}^{N} y(u)
$$

Since CSS descriptors are considered as local features and, hence, do not captures the global shape pertinent to an image contour, so we have decided to resort to two extra global features in a bid to help with the indexing of shapes, namely: circularity and eccentricity. Circularity designates measuring how close a shape in to a circle, which has the minimum circularity measure of $4 \pi$. Circularity is a simple (and hence fast) feature to compute. It is defined as:

$$
\operatorname{cir}=\frac{P^{2}}{A}
$$

With $P$ being the shape perimeter and $A$ its area. As regard eccentricity, it is a global feature that helps measure how shape contour points are scattered around its centroid. It is defined as:

$$
e c c=\sqrt{\frac{\lambda_{\max }}{\lambda_{\min }}}
$$

Where, $\lambda_{\max }$ and $\lambda_{\min }$ are the eigenvalues of the matrix $\mathrm{B}$, such as:

$$
B=\left[\begin{array}{ll}
\mu_{2,0} & \mu_{1,1} \\
\mu_{1,1} & \mu_{0,2}
\end{array}\right]
$$

$\mu_{2,0}, \mu_{1,1}$, and $\mu_{0,2}$ are the shape central moments defined as :

$$
\mu_{p, q}=\sum_{x} \sum_{y}(x-\bar{x})^{p}(y-\bar{y})^{q}
$$

With $\bar{x}$ and $\bar{y}$ representing the coordinates of the shape centroid. The circularity and eccentricity features are the size, rotation and translation invariant.

The descriptors applied as the system shape-indexing means relevant to the present work are, thus, a combination of four-feature sets, namely:

- Circularity feature ( a single global feature)

- Eccentricity feature (a single global feature)

- CSS original-shape descriptors ( $n$ local features, where $n$ depends on the object shape)

- CSS dual-shape descriptors ( $m$ local features, where $m$ depends on the object shape)

\subsection{The shape-feature fuzzification}

In this section, our focus of interest lies in devising fuzzy-shape database. In this context, some efforts have been realized in the previous work (Maghrebi et al. [10], Maghrebi et al.[19]). At this level, our targeted objective consists of moving from crisp shape-feature values for extended CSS descriptors to fuzzy ones. The latter are values comprised between 0 and 1, designing membership degrees to the fuzzy sets. The use of fuzzy features is due to the variations in drawing shape between different users which lead to lack in 
precision of crisp features. Thus, every shape feature turns out to be represented by fuzzy sets which design fuzzy membership functions. For the sake of selecting the appropriate membership functions, a special study of the shape features (concavity and convexity, eccentricity and circularity) has to be undertaken through administrating a database test (see figure 8). The global features' eccentricity and circularity are both presented by three fuzzy sets: Low, Medium and High. While the local features, presented by both concavity and convexity peaks, are respectively annotated by $\left(\mu_{i}, \sigma_{\mathrm{i}}\right)$ and $\left(\mu_{\mathrm{i}}, \sigma_{\mathrm{i}}^{\prime}\right)$, we propose to represent the curvilinear abscissa $\mu_{\mathrm{i}}$ (respectively $\mu_{\mathrm{i}}^{\prime}$ ) by means of two fuzzy sets : Low and High. With regard to the filtering numbers $\sigma_{i}\left(\right.$ respectively $\sigma_{i}{ }^{\prime}$ ), they are decomposed into three fuzzy semantic linguistics terms: Low, Medium and High. We have used trapezoidal functions to represent the different descriptors of shape.

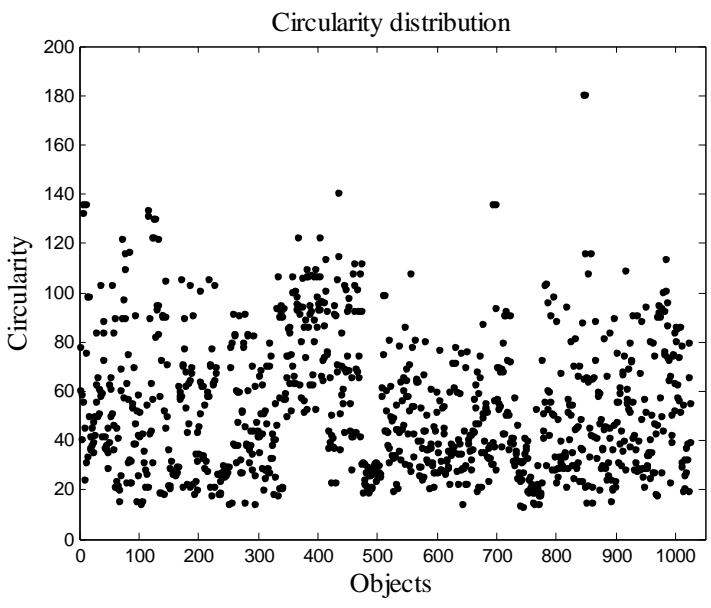

(a)

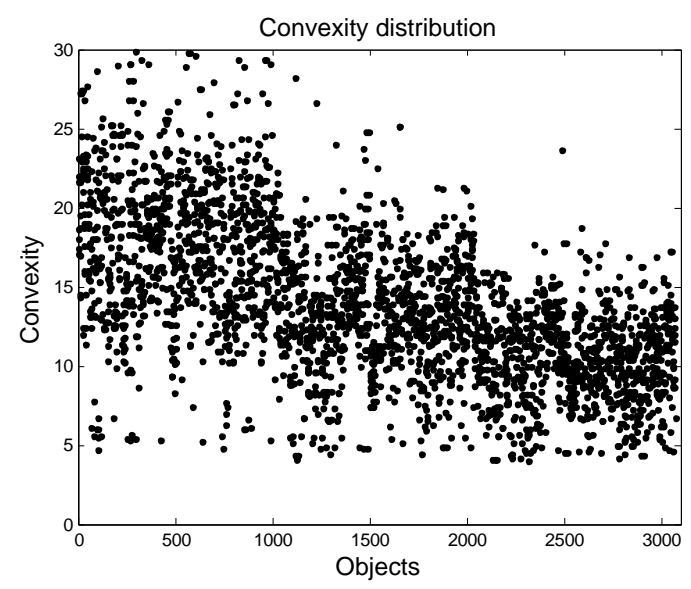

(b)

Figure 8. Samples of shape features distribution: a) Circularity b) Convexity $\sigma$

\section{The retrieval process}

In our previous work (Maghrebi et al. [20]) we have used the semantic color labeling with other highlevel features to attain the high-level query concept. The present work provides a graphic user interface (GUI) whereby an object relevant boundary and/or color(s) can be drawn. The object's query features (shape and/or color) are extracted and fuzzified in such a way as to be compared with the database (DB) object fuzzy features. The use of fuzzy features has its justification in the variations noticed in the drawing shapes as provided by different users, likely to lead to lack of precision as regards the crisp features. As is the case with the recognition system, the similarity measure is very important in matching the two shapes (the query shape and the DB one). It is worth noting that on examining the relevant literature, one can come across various fuzzy-similarity measures proposed (Pappis and Karacapilidis[21], Chen et al.[22], Wang[23], Baccour and Alimi[24]). As far as our retrieval process is concerned, and for the sake of rendering shapes similar to the query proposed ones, we have reckoned it useful to apply Chen et al.[22]) fuzzy similarity measure which severs to express the resemblance degree between two fuzzy sets $R$ and $Q$.

Let us suppose, in this context, that $\mathrm{R}$ and $\mathrm{Q}$ are two fuzzy sets defining, respectively, a fuzzy referencedobject's boundary descriptors (extracted from our DB framework) and a fuzzy query related to the object boundary descriptors; the similarity measure can be defined as:

$$
w_{S}(R, Q)=1-\frac{1}{N} \sum_{i=1}^{N}\left|\mu_{R}\left(x_{i}\right)-\mu_{Q}\left(x_{i}\right)\right|
$$

The equation 13 uses the Hamming distance between the fuzzy membership degrees of a referenced shape $\mu_{R}\left(x_{i}\right)$ (shapes drawn from our proper DB) and the fuzzy membership degrees of the query-submitted shape $\mu_{Q}\left(x_{i}\right)$. So, we undertake to compute the hamming distance $\mathrm{d}_{\mathrm{y}}$, as defined in equation 14 , by in the following way:

$$
d_{y}=\sum_{i=1}^{N_{y}}\left|\mu_{R}\left(x_{i}\right)-\mu_{Q}\left(x_{i}\right)\right|
$$

Then, we turn to compute the hamming distance (Eq.14) pertaining to each shape descriptor, individually which are eccentricity, circularity, concavity and convexity. So, let $d_{e c c}, d_{c i r}, d_{c o n v}$ and $d_{c o n x}$ be the respective 
distances relevant to the object's shape descriptors computed using the equation defined in 14 . Hence, equation 13 turns out to be equivalent to:

$$
w_{S}(R, Q)=1-\frac{1}{N}\left(d_{e c c}+d_{c i r}+d_{\text {conv }}+d_{\text {conx }}\right)
$$

Objects' shapes derived from the database and having fuzzy similarity degrees close to 1 with a submitted drawing query are considered to be similar. In this study, we undertake to select objects which have fuzzy similarity shape degrees (Eq.15) greater than or equal to 0.8. From the results sorted according to their respective distance measure, we have chosen the first ten set of mosaic-images.

For the aim of further increasing the system's precision, we propose to perform fusion procedure between the fuzzy shape and fuzzy color features. Moreover, the user can also propose to choose three colors, the perceptual color(s) which is (are) fuzzified and inferred to obtained fuzzy query color. To match the referenced object's color(s) between and the query object's one, we use the fuzzy membership-distance degree with regard to two similar fuzzy colors applying the formula of Chen et al.[22].

Let the object's query and database color sets be annotated respectively by $C q=\left\{\left(x, \mu_{q}(x)\right\}\right.$ and $C_{r e f}=\left\{\left(x, \mu_{r e f}(x)\right)\right\}$. Then, the fuzzy color similarity measure is defined as follows:

$$
w_{C}\left(C_{q}, C_{r e f}\right)=1-\frac{1}{M} \sum_{i=1}^{M}\left|\mu_{q}\left(x_{i}\right)-\mu_{r e f}\left(x_{i}\right)\right|
$$

If the query combines both color and shape, a weight $\alpha$ has to be applied to the fusion formula combining the two similarity measures, such as:

$$
\text { Global_Sim } \text { object }_{1}=\alpha w_{S}\left(S_{q}, S_{\text {ref }}\right)+(1-\alpha) w_{C}\left(C_{q}, C_{\text {ref }}\right)
$$

Where $\alpha$, is a parameter characterizing the relevance between shape and color. It is in the range of 0 and 1 and determined experimentally. The pertinent value was found $\alpha=0.4$ and will be discussed later. In the following, we give the algorithm of the retrieval process proposed in this work:

-Accept the user hand drawing query shape with color choice,

-Extraction of fuzzy-color naming as indicated in section 4.3

-Definition and fuzzification of query object shape descriptors as indicated in section 5

-For every mosaic-image in the DB Do

--Compute the fuzzy shape similarity $W_{S}(E q .15)$

--Compute the fuzzy color similarity $W_{c}(E q .16)$

--Compute the global similarity Global_sim(Eq.17)

\section{End do}

-Sort results in decreasing order of Global_sim.

-Return relevant mosaic images

\section{Results and discussion}

For experimental purposes, we undertake to test our devised system on a set of 1050 objects extracted from 200 Tunisian historical-mosaic monuments. On a first stage, 100 queries have been applied during the retrieval process. The user can draw a query using a pen or a mouse. Figure 9 shows queries exclusively based on object shape. As seen, the FMIRS system returns relevant results but some errors are remarked. The sources of errors are mainly due to the resemblance between objects boundaries. For example in figure 9.a, the query shape represents a boat contour but the first returned image shows that the drawing query is assimilated to the castle boundary. Also, in figure 9.b the mosaic response (in the bottom at right), the fish contour is confused with the women one.

In addition, the returned results present mosaic-images of different colors. To make our system more accurate, we have added the color descriptors. Now, the idea is to fuse the objects boundary and the color 
features to obtain images containing objects of colors in harmony with their form. The fusion is made by the equation 17. Figure 10 shows the queries that combine both the shape and the color features. In addition to the draw of the query, the user chooses up to three colors. As can be noticed, in Figure10a, light colors have been selected, whereas in Figure10b we have chosen dark ones. One might well observe the variation in the mosaic images provided as a response to the submitted questions exclusively with regards to the object boundary (Figure 9), or by adding the color descriptors (Figure 10). These queries are aimed to test the system's recall and precision performance as well as its ability to accurately handle queries designating the objects' desired shape and/or color.

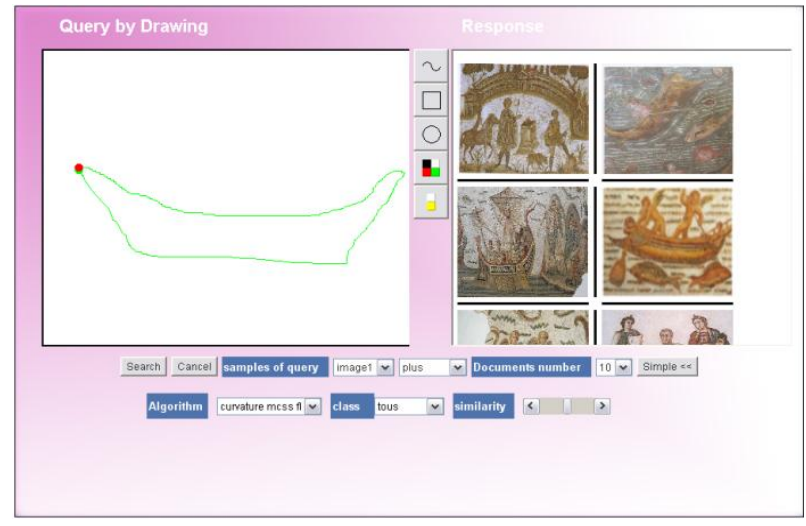

(a)

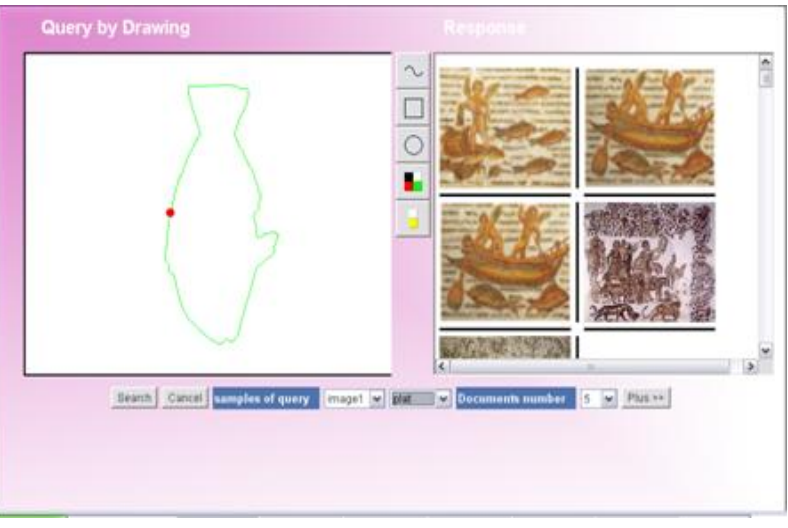

(b)

Figure 9. Samples of a drawing-query type representing a) boat shape and b) fish shape

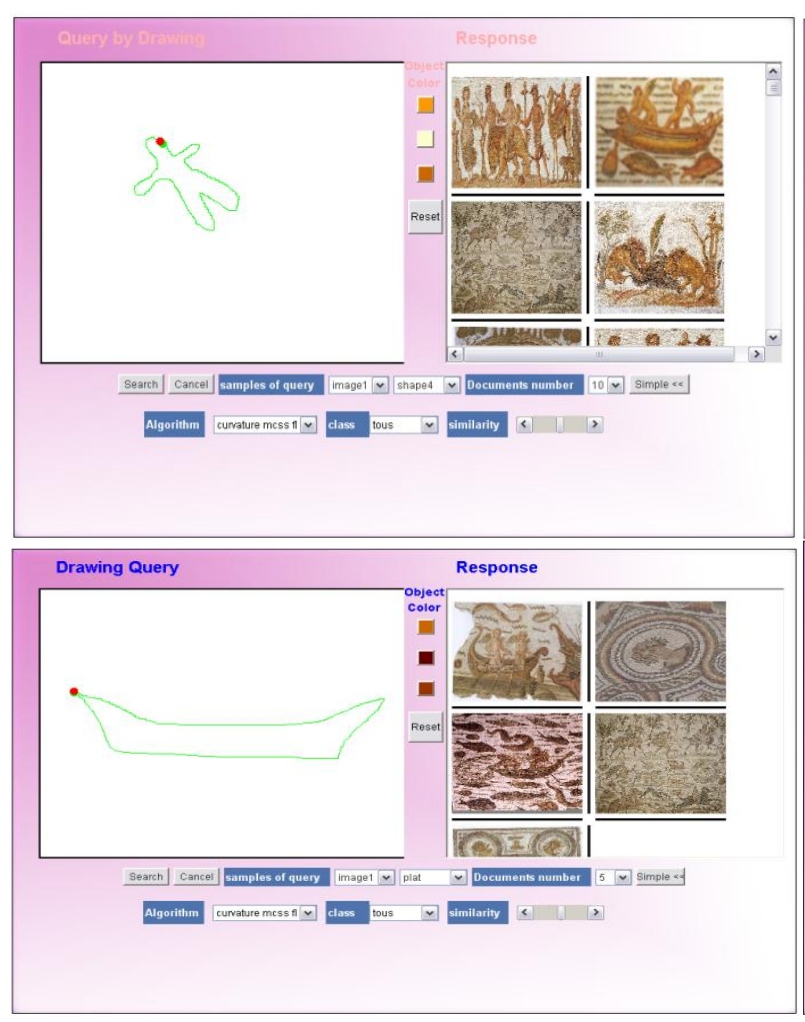

a)

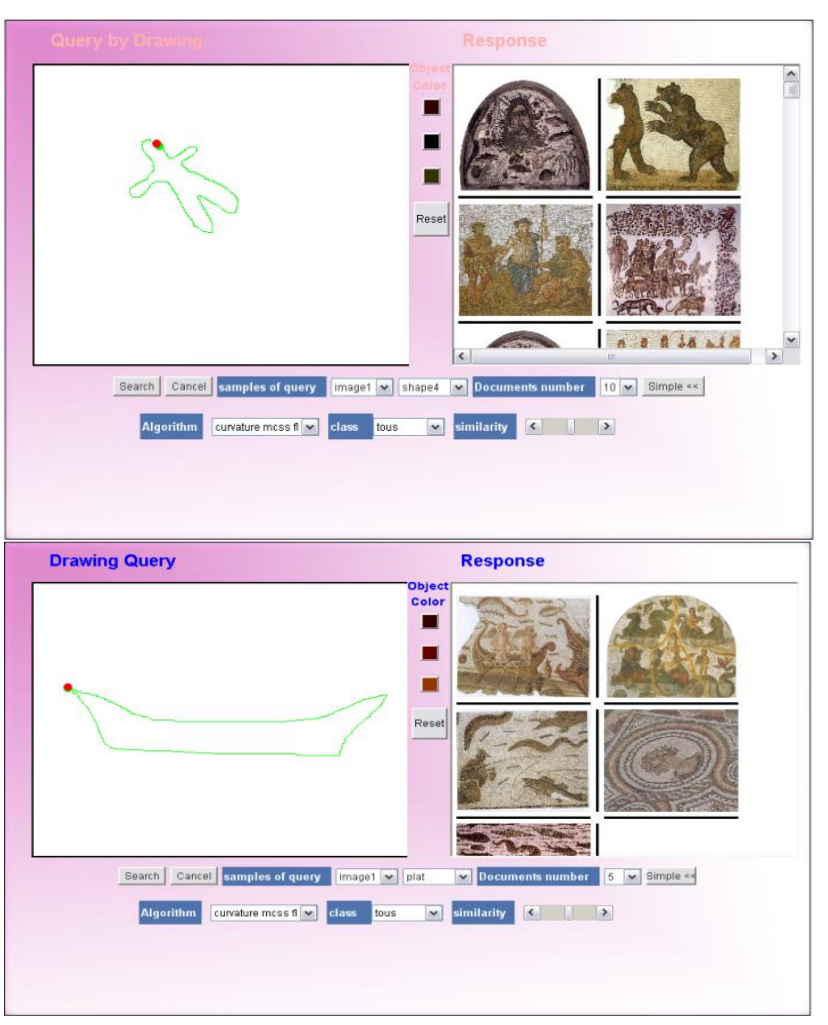

b)

Figure 10. Samples of a drawing-based query fusion with color descriptors representing a person and boat boundaries and response a) light colors b) dark colors

The recall measures the ability of the system to present all relevant images and defined as: 


$$
\text { Recall }=\frac{\text { number of relevant images retrieved }}{\text { number of relevant images in database }}
$$

It is obvious to reach recall of $100 \%$ by returning all relevant images in response to any query. Therefore, recall alone is not sufficient; we need to measure also the number of non-relevant images returned by the system. This can be achieved by computing the so-called precision:

$$
\text { Precision }=\frac{\text { number of relevant images retrieved }}{\text { total number of images retrieved }}
$$

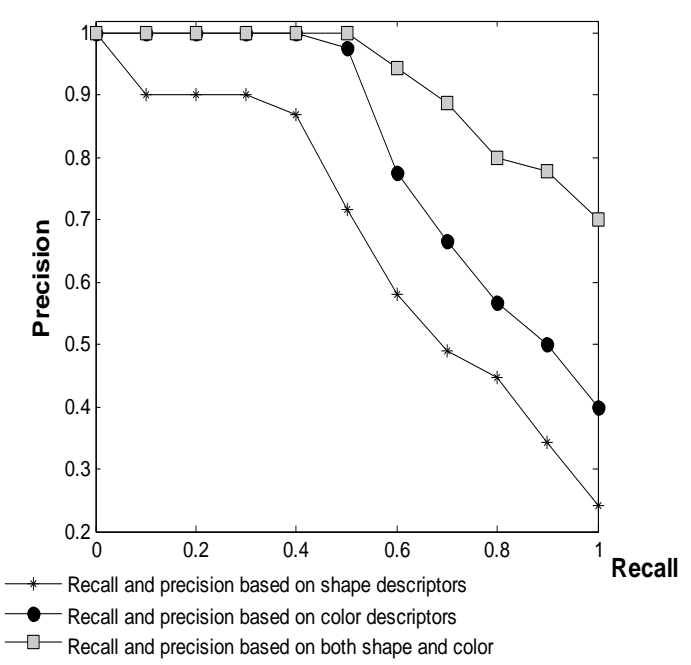

(a)

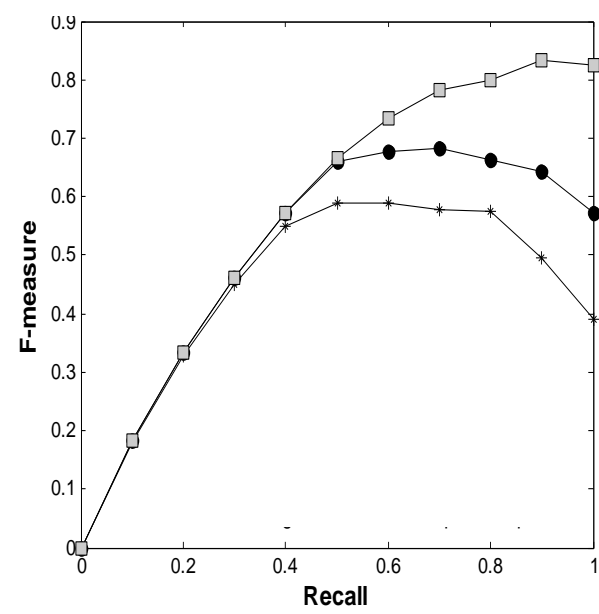

(b)

Figure 11. Comparison between color and shape and their fusion. a) The recall and precision. b) The Fmeasure

Figure 11.a shows the precision as a function of the recall using drawing and/or color queries. The reached results indicate that fusion between the shape and color features increase the system's precision rate compared to the use of exclusively shape or color. The query using a drawing boundary gives acceptable results. While queries based on color gives more acceptable results. Moreover, the fusion between shape and color gives better results.

However, we have been able to achieve overall recall and precision rates of $72.8 \%$ and $43.9 \%$ respectively, regarding the drawing formulated query, whereas fusion relying on the dominant fuzzy colors descriptors helps mainly increase the precision up to $77.35 \%$ and yields an adequate recall rate of $86.28 \%$ computed on the base of 10 first returned images. As for the system's performance and overall recall and precision relevant to the different querying modes, they are summed up in Table 3 for different weights.

Table 3 shows the recall and precision for drawing and color queries and their fusion for different weights $\alpha$. Since recall and precision are inversely related, i.e. when recall increases, precision decreases and inversely, as seen in table $3 \alpha=0.4$ gives acceptable compromise between recall and precision.

In addition, the proposed system is evaluated through the F-measure, undertaken to highlight the harmonic mean between the precision and recall rates, and defined by:

$$
F=\frac{2 . \text { recall } \text {.precision }}{(\text { recall }+ \text { precision })}
$$


Figure 11.b shows the harmonic mean comparison between shape, color as well as both features'. This figure confirms results indicating that fusion improve the system precision.

\begin{tabular}{|c|c|c|c|}
\hline & & \multicolumn{2}{|c|}{ Average } \\
\hline \multicolumn{2}{|l|}{ Query nature } & Recall & Precision \\
\hline \multicolumn{2}{|l|}{ Drawing query } & $72.8 \%$ & $43.9 \%$ \\
\hline \multicolumn{2}{|c|}{ Query using colour features } & $84.83 \%$ & $63.33 \%$ \\
\hline \multirow{5}{*}{ Fusion of colour and shape } & $\alpha=0.3$ & $77.45 \%$ & $51.60 \%$ \\
\hline & $\alpha=0.4$ & $86.28 \%$ & $77.35 \%$ \\
\hline & $\alpha=0.5$ & $76.85 \%$ & $68.14 \%$ \\
\hline & $\alpha=0.6$ & $60.37 \%$ & $78.44 \%$ \\
\hline & $\alpha=0.7$ & $52.15 \%$ & $84.50 \%$ \\
\hline
\end{tabular}

Table3. Average recall and precision of query modes

Finally we have to evaluate our retrieval system by comparison to other results. To our knowledge there is no advanced research about mosaic retrieval system, so we choose to realize a comparison with results of Van den Broek et al.[1] as shown in figure 12. It should be noted that the system proposed by Van den Broek et al.[1] was applied to a database of 1000 colored images and consists on fusion between color, texture and shape. Figure 12.a shows that our system gives slightly better results, but in figure 12.b the recall shows largely better results. The recall defined values start from about $86 \%$ indicating that most relevant images in the database are retrieved in the first 50 returned images. We can conclude that our retrieval system based on shape and color fusion gives largely accepted results.
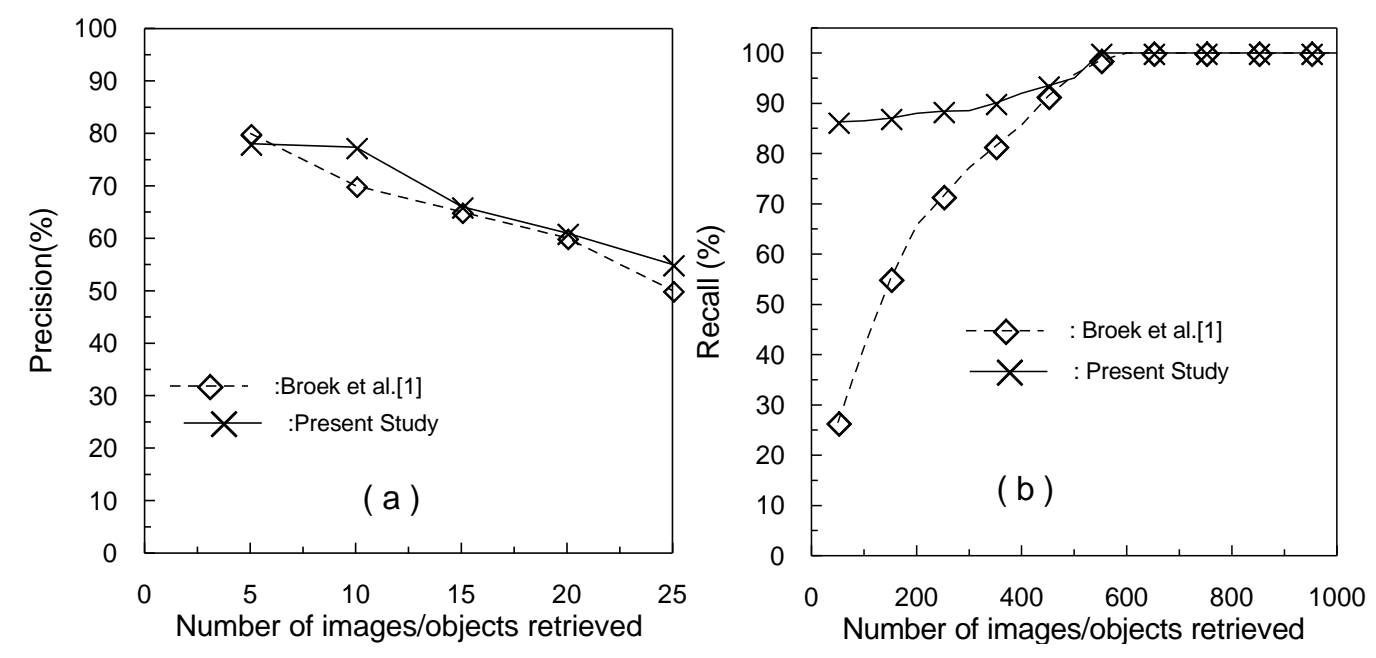

Figure 12. Comparison of average precision (a) and recall (b) between our retrieval system and Van den Broek et al.[1] one.

\section{Conclusion}

In this paper, an image indexing and retrieval of historical mosaics has been advanced. The proposed framework has been conceived to be user-friendly providing a multi-querying mode design. Actually the system has been tested on a database enclosing a set of 1050 objects of 200 very rich images pertinent to Tunisian mosaics. Our system undertakes to index object using fuzzy features. On a first stage, we extract 
the fuzzy features which present the extended CSS shape descriptor, which is robust to scale, rotation and translation. On a second stage, the present work provides a fuzzy color-quantization approach that helps extract and process a semantically-based color labeling. Noteworthy, also, our suggested indexing procedure's novel contribution lays in its ability to undertake, simultaneously, a mutli-querying mode (drawing and/or color(s)). One might also note that the results achieved prove to be very promising, as the queries combining a fusion of both object boundary and colors have yielded average recall and precision $86.28 \%$ and $77.35 \%$ respectively vs. $72.8 \%$ and $43.9 \%$ respectively, for the drawing-based queries. As a prospective for future works, we reckon to go further with improving our fuzzy model's indexing and retrieval capabilities. We suggest introducing other querying mode as the CBIR mode applied on an international historical mosaics' database.

Acknowledgement: The Authors would like to acknowledge the financial support of this work by grants from General Direction of Scientific Research (DGRST), Tunisia, under the ARUB program.

\section{References}

[1]Van den Broek, E.L., Kisters, P.M.F, and Vuurpijl, L.G.: Content-Based image Retrieval Benchmarking Utilizing Color Categories and Color distributions. Journal of imaging science and technology, 49: 293301, 2005.

[2]Van den Broek, E.L., Kok, T., Schouten, T.E. and Vuurpijl, L.G.: Human-Centered Content-Based Image Retrieval. Human Vision and Electronic Imaging XIII, San Jose, USA: SPIE 6806:28-31, 2008

[3]Flickner, M., Sawhney, H., Niblack, W., Ashley, J., Huang, Q., Dom, B., Gorkani, M., Hafner, J., Lee, D., Petkovic, D., Steele, D., and Yanker, P.: Query by Image and Video Content: The QBIC system. IEEE Computer, 28: 23-32, 1995. <http://dx.doi.org/10.1109/2.410146>

[4]Schomaker, L., Vuurpijl, L., and Deleau, E.: New Use for the Pen: Outline-Based Image Queries. Proceedings of the 5th International Conference on Document Analysis and Recognition (ICDAR), Piscataway, New Jersey, USA, 293-296, 1999. <http://dx.doi.org/10.1109/icdar.1999.791782>

[5]Vuupij1, L., Shomaker, L., and Broek, E.: Vind(x): Using The User Through Cooperative Annotation. The $8^{\text {th }}$ International.Workshop. 20 . Frontiers in Handwriting Recognition (IWFHR.8), Canada, 221-225, 2002.

[6]Chang, J.W., and Kim, Y.J.: XML Document Retrieval System Supporting Multimedia Web Service for Digital Museum. IEEE international Conference on Web services, ICWS, Utah, USA. IEEE Press, 10011007, 2007. <http://dx.doi.org/10.1109/icws.2007.198>

[7]Stanco, F., Battiato S., and Gallo, G.: Digital imaging for cultural heritage preservation: analysis, restoration and reconstruction of ancient Artwork. CRC Press Taylor and Francis group, 2011.

[8]M'hedhbi, M., Mezhoud, R., M'hiri, S., and Ghorbel, F.: A new content-based image indexing and retrieval system of mosaic images, ICT-TA'06, 3rd Int. Conf. on Information and Communication Technologies: from Theory to Applications, Damascus, Syria, 1715-1719, 2006.

[9]Maghrebi, W., Borchani, A., Khabou, M.A., and Alimi, A.M.: A System for Historic Document Image Indexing and Retrieval Based on XML Database Conforming to MPEG7 Standard, Lecture Notes in Computer Science, 5046:114-125, 2008.<http://dx.doi.org/10.1007/978-3-540-88188-9_12>

[10]Maghrebi, W., Baccour, L., Khabou, M.A., and Alimi, A.M.: An Indexing and Retrieval System of Historic Art Images Based on Fuzzy Shape Similarity. Lecture Notes in Computer Science, 4827:623-633, 2007.<http://dx.doi.org/10.1007/978-3-540-76631-5_59>

[11]Huang, L.K, and Wang, M.J.: Image thresholding by minimizing the measure of fuzziness. Pattern Recognition, 28:41-51, 1995. <http://dx.doi.org/10.1016/0031-3203(94)e0043-k>

[12]Berk, T., Brownston, L., and Kaufman, A.: A new color-naming system for graphics language. IEEE Computer Graphics Application, 2: 37-44, 1982. <http://dx.doi.org/10.1109/mcg.1982.1674223>

[13]Conway, D.M.: An experimental comparison of three natural language color naming models. Proceedings of the East-West International Conference on Human-Computer Interactions, St. Petersburg, Russia, 328-339, 1992.

[14]Kelly, K.L.: Central Notations for the Revised ISCC-NBS Color-Name Blocks. Journal of Research of the National Bureau of Standards, 61:427-431, 1958. <http://dx.doi.org/10.6028/jres.061.035>

[15]Shamir, L.: Human Perception-based Color Segmentation Using Fuzzy Logic. Int. Conference on Image Processing, Computer Vision and Pattern Recognition, Las Vegas, USA, 496-505, 2006. 
[16]Benavente R., Vanrell M., and Baldrich R.: A Data set of Fuzzy Color Naming. Color Research \& Application, 31(1): 48-56, 2006. <http://dx.doi.org/10.1002/col.20172>

[17]Mokhtarian, F., Abbasi, S., and Kittler, J.: Efficient and robust retrieval by shape through curvature scale space. Proceedings of the first International Workshop on Image Databases and Multimedia Search, Amsterdam, the Netherlands, 35-42, 1996. <http://dx.doi.org/10.1142/9789812797988_0005>

[18]Kopf, S., Haenselmann T., and Effelsberg, W.: Shape-based posture recognition in videos. Proceedings of Electronic Image, 5682:114-124, 2005.

[19]Maghrebi, W., Khabou, M.A., and Alimi, A.M.: A Fuzzy Metadata to Index and Retrieve Images of Roman Mosaics, International Conference on Fuzzy Computation Theory and Applications, Vilamoura, Algarve, Portugal, 233-240, 2013.

[20]Maghrebi, W., Khabou, M.A, Benammar A., and Alimi, A.M.: An Intelligent multi-object retrieval system for historical mosaics. Int.J of Advanced Computer Science and Applications 4:103-110, 2013

[21]Pappis, C., and Karacapilidis, I.: A comparative assessment of measures of similarity of fuzzy values. Fuzzy sets and systems, 56: 171-174, 1993.<http://dx.doi.org/10.1016/0165-0114(93)90141-4>

[22]Chen, S., Yeh, M., and Hsiao, P.: A comparison of similarity measures of fuzzy values. Fuzzy sets and systems. 72: 79-89, 1995. <http://dx.doi.org/10.1016/0165-0114(94)00284-e>

[23]Wang, W.J.: New similarity measures on fuzzy sets and on elements. Fuzzy sets and systems, 85:305309, 1997. <http://dx.doi.org/10.1016/0165-0114(95)00365-7>

[24]Baccour, L., and Alimi, A.M.: Applications and Comparisons of Fuzzy Similarity Measures. WCCI, IEEE World Congress on Computational Intelligence, Barcelona, Spain, 18-23. 2010

$<$ http://dx.doi.org/10.1109/fuzzy.2010.5584276> 